\section{Search for contaminant in EMS outbreak goes slowly}

Tokyo \& Washington. Hopes for a swift and conclusive investigation into the causes of an outbreak in the United States of a fatal blood disorder have foundered on the difficult nature of the problem facing researchers in Japan and the United States.

The outbreak of eosinophilia-myalgia syndrome (EMS), in which at least 27 people died and more than 1,500 were affected in 1989 and 1990, has been traced to consumption of a dietary supplement, L-tryptophan, that is made with the help of a genetically engineered organism. That circumstance has led to questions about the safety of the bioengineering process itself. The case has also drawn unwanted public attention to the manufacturer, Showa Denko, which has given grants of more than $\$ 3$ million to 22 research groups at universities and institutes in the United States, as well as supporting a smaller research effort in Japan.

Progress has nevertheless been slow. Scientists believe it could take several more years to find an answer. And they are not willing to speculate on whether the problem lies in the production process. L-tryptophan is an essential amino acid sold in tablet, capsule and powder form as a food supple- ment. It is metabolized to serotonin, a neurotransmitter, and has been used to treat insomnia, depression and other disorders.

Contrary to initial expectations, it now appears that EMS "is not due to a single cause or the effect of a single contaminant", says Sam Page at the US Food and Drug

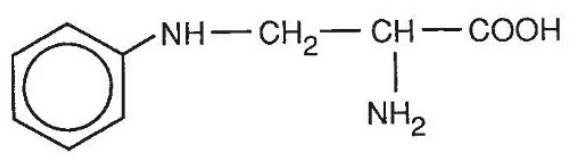

The latest candidate cause of EMS

Administration. "We're looking at an enormously complex inflammatory disease process." There are some 60 contaminants in L-tryptophan, and even pure L-tryptophan appears to cause EMS-like symptoms in some people.

In 1990, when scientists at Minnesota Department of Health announced that batches of suspect L-tryptophan associated with the EMS outbreak were made with the help of a genetically engineered strain of Bacillus amyloliquefaciens, opponents of recombinant-DNA technology used the discovery to question the safety of all genetically engineered products (see Nature 346, $787 ; 1990)$. A cloud has been hanging over the biotechnology industry ever since.

Although there is still no answer to the question of whether genetic engineering played a role in the epidemic, the delay in answering that question appears to be due more to the pace of science than to a wish to suppress the truth. Contrary to the allegations of some activists and EMS victims, the reason there is still no known cause of the outbreak "is more a technical problem than a coverup," says Vincent Falanga, a University of Miami researcher who is investigating L-tryptophan's molecular mechanisms. Most researchers say that Showa Denko has been cooperative in supplying data on the production processes and samples of the material, and scientists funded by the company say they have been free to publish their findings. Others point out, however, that most of the research funded by Showa Denko concerns basic biomedical questions about L-tryptophan rather than the exact cause of the contaminations.

For the past two years, researchers have focused on one contaminant (peak E) found in liquid chromatographs of the batches of L-tryptophan associated with EMS. But extensive animal tests by researchers at the US National Institutes of Health have failed to establish more than a partial link between this contaminant and EMS.

Last month, a group of US scientists and Legal battles amid the science

Washington. US government scientists trying to track down the cause of EMS must deal with more than just scientific issues. Attorneys for Showa Denko, the Japanese company that produced the L-tryptophan implicated in an outbreak of the disease, have gone to great lengths to obtain the most recent results of government research into the disease. Some researchers say that their behaviour has had a chilling effect on independent investigations.

Their aggressive legal tactics are understandable, given that Showa Denko has been sued for more than a billion dollars by EMS sufferers. But to Esther Sternberg, an EMS researcher at the US National Institute of Mental Health (NIMH) who was the focus of one such skirmish, the company's efforts to acquire her pre-publication data crossed the line from legitimate inquiry to intimidation.

In late 1990, attorneys from Showa Denko's US law firm, Cleary, Gottlieb, Steen and Hamilton, filed several requests under the Freedom of Information Act (FOIA) for Sternberg's pre-publication data on EMS and L-tryptophan. Sternberg supplied some of the data on which her published work was based, but she balked at a request for her glass pathology slides and protocols of planned experiments. After reviewing the case, officials from NIMH and other federal agencies that were the subject of similar FOIA requests decided that government scientists were not required to make their data available before publication. So the Showa Denko lawyers wrote to Sternberg herself. On the advice of her attorney, she refused to respond. Company lawyers also called Sternberg's boss, the director of the NIMH, and tried unsuccessfully to get Sternberg to meet them.

A congressional panel is looking into these legal manoeuvres as part of its continuing investigation into the L-tryptophan case. One aspect of the inquiry, by the human resources subcommittee of the House of Representatives Committee on Government Operations, is focused on whether Showa Denko's actions have hindered government investigations into the outbreak. another group from Japan independently announced the chemical structure of a second contaminant in the suspect L-tryptophan that they think may be the cause of the EMS outbreak. The new contaminant was found by researchers at the Mayo Clinic in the United States and by another group at the National Institute of Hygienic Sciences in Tokyo and has been identified as 3 phenylamino-L-aniline. This chemical is very similar to the contaminant in cooking oil that caused an outbreak of EMS in Spain in 1981.

But further animal experiments are needed to determine if the new contaminant is the cause of EMS, and nothing is known about the pathway of formation of the new contaminant in the process of production of $\mathrm{L}$ tryptophan. However, this has not stopped some Japanese newspapers from speculating that the contaminant is directly linked to the use of a genetically engineered organism.

Mitsuru Uchiyama, director-general of the National Institute of Hygienic Sciences and leader of the Japanese group that isolated the new contaminant, hopes to collaborate with Page in establishing the pathway of formation of the new contaminant. But researchers in both countries expect that it will take several more years to pin down the cause of EMS.

David Swinbanks \& Christopher Anderson 